\title{
Vascular endothelial growth factor C expression correlates with tumour metastatic recurrence in patients with oesophageal squamous cancer after Ivor-Lewis oesophagectomy
}

\author{
Zhi-Gang Sun ${ }^{1,2}$, Zhou Wang ${ }^{1}$, Li-Mei Ma ${ }^{1}$, Min Zhang ${ }^{3}$, Gang Chen ${ }^{1}$, Xiang-Yan Liu \\ 1Department of Thoracic Surgery, Provincial Hospital Affiliated to Shandong University, Jinan, China \\ 2Department of Thoracic Surgery, Jinan Center Hospital Affiliated to Shandong University, Jinan, China \\ 3Department of Dermatology, Jinan Center Hospital Affiliated to Shandong University, Jinan, China
}

Przegląd Gastroenterologiczny 2012; 7 (1): 31-37

DOI: $10.5114 /$ pg.2012.27220

Key words: oesophageal squamous cell cancer, recurrence, vascular endothelial growth factor C.

Address for correspondence: Zhou Wang MD, Department of Thoracic Surgery, Provincial Hospital Affiliated to Shandong University, Jinan 250021, China, phone: (0086)13665312567, e-mail: wangzhouszg@126.com, Li-Mei Ma, Department of Thoracic Surgery, Provincial Hospital Affiliated to Shandong University, Jinan 250021, China, phone: (0086)15168863715, e-mail: malm92@126.com

\begin{abstract}
Introduction: Lymph node status is one of the most important prognostic factors for oesophageal squamous cell carcinoma (ESCC). Recent studies have revealed that several genes and molecules are involved in the origin and progression of ESCC. Tumour progression and metastasis require angiogenesis. It has recently been reported that vascular endothelial growth factor C (VEGF-C) induces not only angiogenesis but also lymphangiogenesis via VEGF receptor-2 and VEGF receptor-3.

Aim: To investigate the correlation between VEGF-C expression and tumour metastatic recurrence in patients with ESCC. Material and methods: Ninety-two ESCC specimens were reevaluated by reverse transcriptase-polymerase chain reaction (RT-PCR) to detect VEGF-C mRNA. The correlation between VEGF-C expression and clinicopathological factors was analysed using Fisher's exact probability test or $\chi^{2}$ test. The log-
\end{abstract}

\section{Introduction}

Oesophageal squamous cell carcinoma (ESCC) is one of the deadliest malignancies worldwide. Up to now, radical resection has remained the most effective means of cure for ESCC. However; the long-term outcome after routine oesophagectomy is far from satisfactory, with 5-year survival rates at 20-30\% [1, 2]. Lymph node status is one of the most important prognostic factors for oesophageal cancer. Upon presentation with dysphagia, over $70 \%$ of patients will already have lymph node metastasis [3]. Of those who survive surgery, $80 \%$ will eventually die rank test was used to calculate the recurrence difference. And Cox regression multivariate analysis was performed to determine independent prognostic factors.

Results: The VEGF-C mRNA expression was correlated with $\mathrm{pT}$, $\mathrm{pN}, \mathrm{pTNM}$ and lymphatic invasion. The 5-year tumour metastatic recurrence rate in patients after operation was significantly associated with pT, pN, pTNM stage and VEGF-C mRNA expression. The 5-year tumour metastatic recurrence rate of patients with VEGF-C mRNA expression in oesophageal cancer tissues was significantly higher than that of the patients without VEGF-C mRNA expression. The results of Cox regression multivariate analysis confirmed that $\mathrm{PT}$ status and VEGF$\mathrm{C}$ mRNA expression were independent relevant factors.

Conclusions: The VEGF-C expression is related to tumour invasion, lymphatic invasion, lymph node metastasis, tumour stage and tumour metastatic recurrence in ESCC patients.

from tumour recurrence; and at least $40 \%$ of these are due to recurrence in lymph nodes [4]. However, the precise mechanisms that underlie the development and progression of ESCC are far from clear. To date, no biomarkers of ESCC have been proposed [5].

Recent studies have revealed that several genes and molecules are involved in the origin and progression of oesophageal cancer. Tumour progression and metastasis require angiogenesis, which is induced by various factors including the vascular endothelial growth factor (VEGF) family of polypeptide growth factors [4]. It has recently been reported that vascular endothelial growth 
factor C (VEGF-C) induces not only angiogenesis but also lymphangiogenesis via VEGF receptor-2 and VEGF receptor-3 [5]. Previous reports have shown that VEGF-C expression in cancer tissues has a positive correlation with the risk of lymphatic metastasis in a variety of cancers. A similar tendency has been reported for oesophageal cancers [4-6]. However, little is known about the correlation between VEGF-C expression and tumour metastatic recurrence in patients with ESCC.

\section{Aim}

The present study was therefore designed to investigate the risk of tumour metastatic recurrence in patients with ESCC after Ivor-Lewis oesophagectomy, based on the detection of VEGF-C mRNA.

\section{Material and methods Patients}

There were 278 patients with mid-thoracic ESCC who underwent resection in our department from January 2001 to January 2005. Among them there were 92 non-consecutive patients with ESCC of the middle thoracic oesophagus who underwent Ivor-Lewis oesophagectomy with two-field lymph node dissection [7, 8]. The latter patients were enrolled in this study. The inclusion criteria are as follows: (1) squamous cell carcinoma of the middle thoracic oesophagus pathologically diagnosed as postoperative pathological stage I-III, (2) no preoperative radiotherapy or chemotherapy, (3) patients were otherwise healthy and without surgical contraindication, (4) no evidence of tumescent cervical or supraclavicular lymph node disease was noted on physical examination, and a preoperative computed tomography (CT) scan or cervical B ultrasound study indicated no cervical or supraclavicular lymph node metastasis, (5) postsurgical pathology studies proved no residual malignant cell on the upper and lower incisal edges.

Patients who underwent incomplete or no resection were excluded to eliminate the potential influence of residual or extensive unresectable disease. The study group consisted of 75 men and 17 women, ranging in age from 45 years to 72 years. On the basis of the TNM classification of the International Union against Cancer (UICC) from 1997, 4 patients had pT1 disease, 32 patients had pT2 disease, 51 patients had pT 3 disease, 5 patients had pT4 disease; and 22 patients had lymph node metastases. Patients were routinely examined during the first 5 years. The position and time of recurrent disease were recorded completely. The clinicopathological characteristics of the 92 patients are listed in Table I.

\section{Samples}

Ninety-two oesophageal cancer specimens were obtained from the 92 patients. Each specimen was partitioned into two portions. An oesophageal cancer tissue specimen of at least $0.5 \mathrm{~cm} \times 0.5 \mathrm{~cm} \times 0.5 \mathrm{~cm}$ was used as the experimental specimen. The other oesophageal cancer tissue specimen was fixed in $10 \%$ formaldehyde solution for histopathological examination. Histological examination confirmed that all of the cancer tissues studied were squamous cell carcinomas. All the experimental specimens were handled with a fresh set of clean instruments to prevent cross-contamination of VEGF-C mRNA. Each oesophageal cancer tissue was labelled, and then was wrapped quickly in foil and snap frozen in liquid nitrogen for $1 \mathrm{~min}$ and kept at $-80^{\circ} \mathrm{C}$ until RNA extraction.

Total RNA was extracted from each specimen with the Trizol one-step procedure according to the protocol provided by the manufacturer. RNA purity and concentration were determined by standard UV spectrophotometric assay. Primers were designed according to previous reports [9], as follows: 5'-end primer: 5'-AAGGAGGCTGGCAACATAAC-3', 3'-end primer: 5'-CCACATCTGTAGACGGACAC-3'. The primers of VEGFC yielded a 206 base pair (bp) product. The primers of $\beta$-actin yielded a 644 bp product as follows: 5'-end primer: 5'-ACGTTATGGATGATGATATCGC-3', 3'-end primer: 5'-CTTAATGTCACGCACGATTTCC-3'.

Following an initial denaturation at $94^{\circ} \mathrm{C}$ for $5 \mathrm{~min}$, the samples were amplified by 30 cycles of denaturation at $94^{\circ} \mathrm{C}$ for $30 \mathrm{~s}$, annealing at $58^{\circ} \mathrm{C}$ for $30 \mathrm{~s}$, extension at $72^{\circ} \mathrm{C}$ for $30 \mathrm{~s}$, and ended by extension at $72^{\circ} \mathrm{C}$ for $10 \mathrm{~min}$. Polymerase chain reaction products were visualized by electrophoresis through $1 \%$ agarose gels stained with ethidium bromide. Gel images were obtained using AlphalmagerTM 2200 UV-image analyser (Alpha Innotech Corp., USA). The ratios of VEGF-C/ $\beta$-actin were used to semiquantify the levels of VEGF-C.

\section{Adjuvant therapies}

In China, up until now there have been no generally accepted guidelines on standard postoperative adjuvant therapy in the treatment of patients with oesophageal carcinoma. In our department, the indications for adjuvant treatment are often dependent on tumour stage, doctors' habits, and patients' willingness or economic status. In general, we advise patients with pT3-4 to receive radiotherapy and those with $\mathrm{pN} 1$ to receive chemotherapy at least. In our cases, 45 patients received postoperative radiotherapy alone. Postoperative chemotherapy was given to 11 patients in more than three cycles (mainly 5-fluorouracil and cisplatin/carboplatin), and 11 patients received combined chemoradiotherapy. 
Table I. Correlation between VEGF-C mRNA expression, 5-year tumour metastatic recurrence rate and clinical features of the 92 patients with ESCC

\begin{tabular}{|c|c|c|c|c|c|c|c|}
\hline \multirow[t]{3}{*}{ Clinical characteristics } & \multirow[t]{3}{*}{$\begin{array}{l}\text { Patients } \\
N=92\end{array}$} & \multicolumn{3}{|c|}{$\begin{array}{l}\text { VEGF-mRNA } \\
\text { expression }\end{array}$} & \multicolumn{3}{|c|}{$\begin{array}{l}\text { Tumour metastatic } \\
\text { recurrence rate [\%] }\end{array}$} \\
\hline & & $(-)$ & $(+)$ & Value of $p^{a}$ & Patients & Rate [\%] & Value of $p^{b}$ \\
\hline & & 36 & 56 & & 65 & 70.7 & \\
\hline Gender & & & & $0.583^{*}$ & & & 0.941 \\
\hline Male & 75 & 28 & 47 & & 53 & 70.7 & \\
\hline Female & 17 & 8 & 9 & & 12 & 70.6 & \\
\hline Age [years] & & & & $0.197^{\star}$ & & & 0.324 \\
\hline$\geq 60$ & 72 & 31 & 41 & & 49 & 69.1 & \\
\hline$<60$ & 20 & 5 & 15 & & 16 & 80 & \\
\hline Smoking & & & & ${ }^{*} 0.760$ & & & 0.463 \\
\hline- & 13 & 6 & 7 & & 10 & 69.9 & \\
\hline+ & 79 & 30 & 49 & & 51 & 69.5 & \\
\hline Weight loss [kg] & & & & $0.130^{*}$ & & & 0.281 \\
\hline$\leq 5$ & 71 & 31 & 40 & & 49 & 69.0 & \\
\hline$>5$ & 21 & 5 & 16 & & 16 & 72.2 & \\
\hline Tumour length $[\mathrm{cm}]$ & & & & $>0.05^{*}$ & & & 0.456 \\
\hline$<5$ & 17 & 9 & 8 & & 10 & 48.2 & \\
\hline $3-5$ & 61 & 23 & 38 & & 44 & 72.1 & \\
\hline$>5$ & 14 & 4 & 10 & & 11 & 78.6 & \\
\hline Lymphatic invasion & & & & 0.000 & & & 0.124 \\
\hline- & 37 & 25 & 12 & & 25 & 67.6 & \\
\hline+ & 55 & 11 & 44 & & 40 & 72.7 & \\
\hline Vascular invasion & & & & 1.000 & & & 0.170 \\
\hline- & 44 & 17 & 27 & & 29 & 65.9 & \\
\hline+ & 48 & 19 & 29 & & 36 & 75.0 & \\
\hline Differentiation & & & & $>0.05^{*}$ & & & 0.265 \\
\hline Well & 9 & 5 & 4 & & 5 & 55.6 & \\
\hline Moderately & 64 & 23 & 41 & & 46 & 71.9 & \\
\hline Poorly & 19 & 8 & 11 & & 14 & 73.7 & \\
\hline pT & 4 & & & $<0.01^{*}$ & & & 0.000 \\
\hline $\mathrm{T} 1$ & 4 & 4 & 0 & & 0 & 0.0 & \\
\hline $\mathrm{T} 2$ & 32 & 16 & 16 & & 21 & 65.6 & \\
\hline T3 & 51 & 16 & 35 & & 12 & 76.5 & \\
\hline T4 & 5 & 0 & 5 & & 0 & 100 & \\
\hline $\mathrm{pN}$ & & & & $0.025^{*}$ & & & 0.001 \\
\hline- & 70 & 32 & 38 & & 45 & 64.3 & \\
\hline+ & 22 & 4 & 18 & & 20 & 90.9 & \\
\hline PTNM & & & & $<{ }^{*} 0.01$ & & & 0.000 \\
\hline 1 & 4 & 4 & 0 & & 0 & 0 & \\
\hline Ila & 66 & 28 & 38 & & 45 & 68.2 & \\
\hline $\mathrm{IIb}$ & 10 & 4 & 6 & & 8 & 80 & \\
\hline III & 12 & 0 & 12 & & 12 & 100 & \\
\hline VEGF-C mRNA expression & & & & & & & 0.000 \\
\hline- & 36 & - & - & & 18 & 50.0 & \\
\hline+ & 56 & - & - & & 49 & 83.9 & \\
\hline
\end{tabular}

Value of $p^{a}: \chi^{2}$ test, ${ }^{*}$ Fisher's exact probability test, value of $p^{b}$ : Log-rank test 


\section{Follow-up}

None of the patients suffered major perioperative complications and all were discharged from the hospital. Patients were routinely examined every 3 to 6 months during the first 3 years and every 6 months or annually thereafter. During each follow-up visit, the patient underwent a thorough physical examination, chest roentgenography, ultrasonography of the neck and abdomen, chest CT, and endoscopic examination. Some patients even underwent positron emission tomography combined with computed tomography (PET/CT) examination. The location and time of tumour relapse were recorded.

\section{Statistical analysis}

Correlations between VEGF-C expression and clinicopathological factors was analysed using Fisher's exact probability test or $\chi^{2}$ test. The Kaplan-Meier method was used to calculate the survival rate and tumour metastatic recurrence rate. The log-rank test was performed to compare the recurrence difference. Cox regression multivariate analysis was performed to judge independent prognostic factors. Differences were considered significant when the $p$ value was less than 0.05. The statistical data were obtained using an SPSS software package (SPSS 13.0 Inc., Chicago, IL, USA).

\section{Results}

\section{Correlation between VEGF-C mRNA expression and clinical characteristics}

The VEGF-C mRNA was identified in ESCC tissues from 56 patients. The diagnostic sensitivity was $60.1 \%$ (56/92) (Figure 1). The relationship between VEGF-C mRNA and clinicopathological features is shown in Table I. The VEGF-C expression correlated with $\mathrm{pT}(p<$ $0.01), \mathrm{pN}(p=0.025)$, pTNM $(p<0.01)$ and lymphatic

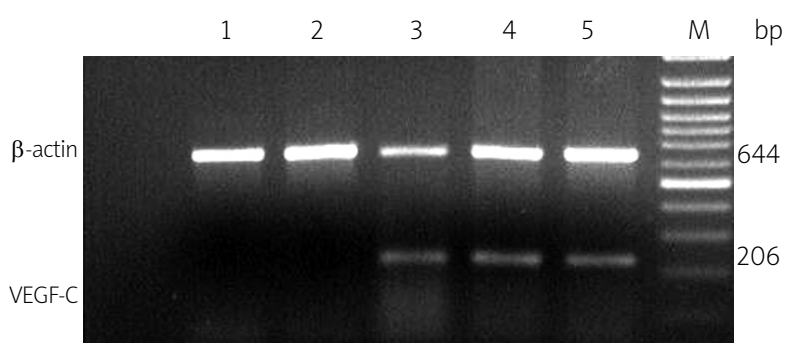

Fig. 1. Expression of VEGF-C mRNA detected by polymerase chain reaction (PCR). Lane 1: corresponding adjacent normal epithelium tissues VEGF-C mRNA (-); lane 2: cancer tissues VEGF-C mRNA (-); lane 3-5: cancer tissues VEGF-C mRNA (+). M molecular marker (bp) invasion ( $p<0.001)$. No other clinicopathological parameter was related to VEGF-C mRNA expression (Table I).

\section{Pattern of recurrence}

Of the 92 patients in this study, recurrence was recognized in 65 patients $(70.7 \%)$ in the first 5 years after operation. Of these, 53 were men and 12 were women, and the median age was 58 years (range: $42-69$ years). The distribution of the sites of tumour recurrence is shown in Table II, and the relationship between clinicopathological factors and recurrent disease is depicted in Table I. Thirtyfour patients (52.3\%) developed lymph nodes recurrence; 21 patients $(32.3 \%)$ developed a haematogenous recurrence, including 10 patients (15.4\%) with lymph nodes and haematogenous recurrence.

\section{Correlation between VEGF-C mRNA expression and tumour metastatic recurrence}

Follow-up data were available for all patients. The median follow-up of the patients was 44 months overall (range: 12 to 164 months). The Kaplan-Meier method indicated that the 5 -year survival rate of the 92 patients was $34.8 \%$. In univariate analysis by the log-rank test (Table III), the 5-year tumour metastatic recurrence rate in patients after operation was significantly associated with pT $(p<0.001)$, pN $(p=0.001)$, pTNM stage $(p<0.001)$ and VEGF-C mRNA expression $(p<0.001)$. The 5 -year tumour metastatic recurrence rate of the patients with VEGF-C mRNA expression in oesophageal cancer tissues was significantly higher than that of the patients without VEGF-C mRNA expression (83.9\% vs. 50\%; $p<0.001$ ) (Figure 2). The results of Cox regression multivariate analysis confirmed that $\mathrm{pT}$ status and VEGF-C mRNA expression were independent relevant factors (Table III).

\section{Discussion}

Metastasis is a complex process that consists of several steps, including dissemination of tumour cells from primary sites, transportation of tumour cells within the lymphatics, settlement of tumour cells within lymph nodes and re-growth of tumours into a detectable size. Although the real mechanism of VEGF-C in tumour metastasis is still unclear, some studies have raised the possibility that VEGF-C might increase metastasis by increasing the number and size of lymphatic vessels, or alternatively by altering the functional properties of existing lymphatics [10]. Several reports have described a significant correlation between VEGF-C expression, tumour lymphangiogenesis, and lymph node metastasis in some cancers [4-6]. Noda et al. [11] reported that 
VEGF-C expression was an independent risk factor for local recurrence of rectal carcinoma, and patients with VEGF-C positive tumours had a significantly worse prognosis than those with VEGF-C negative tumours. So what about the relation of VEGF-C expression with recurrence of ESCC? To test this hypothesis, this study was undertaken to investigate the relationship between

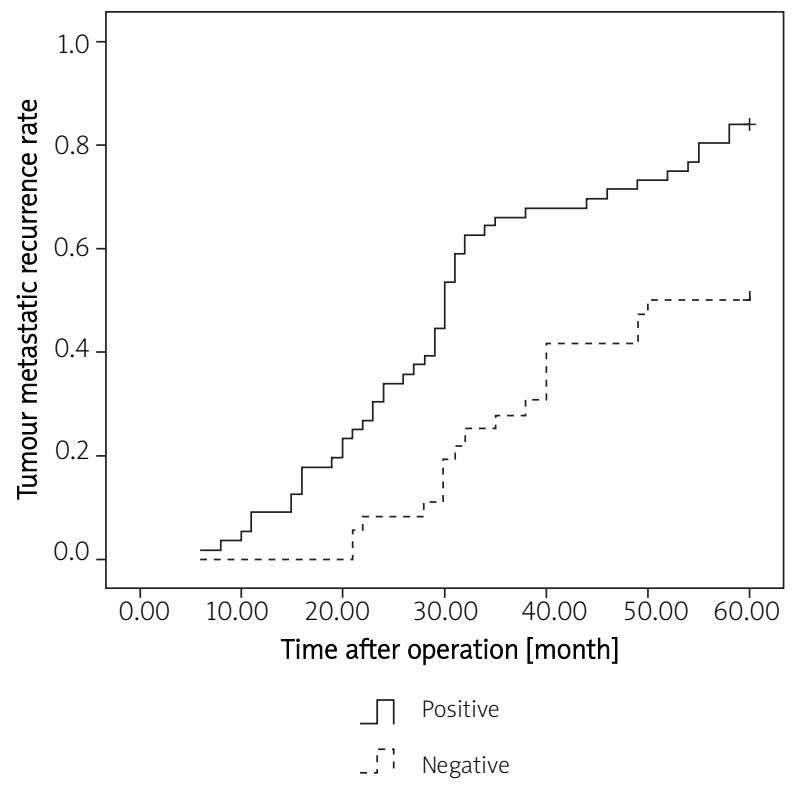

Fig. 2. Kaplan-Meier analysis of the tumour metastatic recurrence rate after operation in patients with negative and positive expression of VEGF-C mRNA, respectively (50\% vs. $83.9 \%$; $p<0.001)$.
VEGF-C expression and postoperative early recurrence in patients with ESCC.

Various authors have reported VEGF-C expression using immunohistochemical staining or reverse transcription polymerase chain reaction in various cancers of the central nervous system [12], head and neck [13], thyroid [14], lung [15], breast [16], stomach [17], large bowel

Table II. Sites of tumour metastatic recurrence in 65 patients

\begin{tabular}{lc} 
Sites of lymph node recurrence & No. of patients (\%) \\
\hline Locoregional recurrence & $34(34 / 65)(52.3)$ \\
\hline Cervical/supraclavicular lymph node & $6(6 / 34)(17.6)$ \\
\hline Mediastinal lymph node & $21(21 / 34)(61.8)$ \\
\hline Abdominal lymph node & $3(3 / 34)(8.8)$ \\
\hline Multiple lymph nodes & $4(4 / 34)(11.8)$ \\
\hline Haematogenous recurrence & $21(21 / 65)(32.3)$ \\
\hline Brain & $1(1 / 21)(4.8)$ \\
\hline Lung & $4(4 / 21)(19.0)$ \\
\hline Oesophagus & $3(3 / 21)(14.3)$ \\
\hline Liver & $9(9 / 21)(42.9)$ \\
\hline Bone & $4(4 / 21)(19.0)$ \\
\hline \begin{tabular}{l} 
Locoregional and haematogenous \\
\hline Liver and abdominal lymph node
\end{tabular} & $10(10 / 65)(15.4)$ \\
\hline Lung and mediastinal lymph node & $4(4 / 10)(40.0)$ \\
\hline \begin{tabular}{l} 
Lung, pleura and mediastinal lymph node \\
\hline Brain, liver and abdominal lymph node
\end{tabular} & $1(1 / 10)(10.0)$ \\
\hline \begin{tabular}{l} 
Liver, lung and mediastinal lymph node \\
\hline Bone, liver and abdominal lymph node
\end{tabular} & $1(1 / 10)(10.0)$ \\
\hline $\begin{array}{l}\text { aMediastinal and cervical lymph node recurrence in } 2 \text { patients; } \\
\text { mediastinal and abdominal lymph node recurrence in } 2 \text { patients }\end{array}$ \\
\hline
\end{tabular}

Table III. Univariate and multivariate analysis with respect to 5-year tumour metastatic recurrence survival

\begin{tabular}{|c|c|c|c|c|c|}
\hline \multirow[t]{2}{*}{ Variable } & \multirow[t]{2}{*}{ Comparison } & \multirow{2}{*}{$\frac{\text { Univariate }}{p^{\star}}$} & \multicolumn{3}{|c|}{ Multivariate } \\
\hline & & & $p$ & HR & $95 \% \mathrm{Cl}$ \\
\hline Gender & Male : female & 0.941 & 0.646 & 1.425 & $0.315-6.438$ \\
\hline Age [years] & $\geq 60:<60$ & 0.324 & 0.212 & 1.534 & $0.783-3.002$ \\
\hline Smoking & Negative : positive & 0.463 & 0.345 & 0.458 & $0.0090-2.320$ \\
\hline Weight loss [kg] & $<5: \geq 5$ & 0.281 & 0.202 & 0.572 & $0.242-1.350$ \\
\hline Tumour length $[\mathrm{cm}]$ & $<5: 5-5:>5$ & 0.456 & 0.857 & 1.055 & $0.589-1.891$ \\
\hline Lymphatic invasion & Negative : positive & 0.124 & 0.552 & 0.807 & $0.398-1.636$ \\
\hline Vascular invasion & Negative : positive & 0.170 & 0.381 & 1.275 & $0.74-12.193$ \\
\hline Differentiation & Well : moderately: poorly & 0.265 & 0.290 & 1.317 & $0.791-2.194$ \\
\hline pT & $\mathrm{T} 1: \mathrm{T} 2: \mathrm{T} 3: \mathrm{T} 4$ & 0.000 & 0.038 & 2.172 & $1.044-4.520$ \\
\hline $\mathrm{pN}$ & Negative : positive & 0.001 & 0.593 & 0.521 & $0.048-5.671$ \\
\hline PTNM & I: Ila : \|b: III & 0.000 & 0.149 & 2.917 & $0.680-12.504$ \\
\hline VEGF-C mRNA & Negative : positive & 0.000 & 0.040 & 2.225 & $1.036-4.778$ \\
\hline
\end{tabular}

$P^{*}$ - log-rank test, $\mathrm{HR}$ - hazard ratio, $\mathrm{Cl}$ - confidence interval 
[18], uterus [19], prostate [20] and oesophageal cancer $[4,5]$. In previous studies, $38.9-76.5 \%$ of patients with oesophageal cancer were shown by immunohistochemistry to express VEGF-C [21]. In this study, we observed VEGF-C expression using RT-PCR, and the results showed that $60.1 \%$ of cases of tumour tissues with ESCC had expression of VEGF-C mRNA. VEGF-C mRNA expression in tumour issues was significantly associated with pT, pN, pTNM and lymphatic invasion. The results of the present study did support previous reports suggesting that VEGF-C plays a clinicopathological role in ESCC.

Some clinical studies have revealed a correlation between VEGF-C expression and tumour recurrence. Noda et al. [11] reported that VEGF-C expression was an independent risk factor for the local recurrence of rectal carcinoma. Chen et al. [22] claimed that VEGF-C expression was high in N2 lung cancer, showing a significant correlation with postoperative early recurrence. There has been only one study published in PubMed that investigated the correlation between VEGF-C expression and tumour recurrence in patients with oesophageal cancer. Kimura et al. [23] reported that the preoperative serum VEGF-C level in patients with oesophageal cancer was significantly higher than in healthy volunteers. Furthermore, patients with recurrence had significantly higher preoperative serum VEGF-C levels than patients without recurrence. In this study, the 5-year tumour metastatic recurrence rate of the patients with VEGF-C mRNA expression in oesophageal cancer tissues was significantly higher than that of the patients without VEGF-C mRNA expression. To eliminate the impact of mixed factors correlated with prognosis on statistical analysis, Cox regression multivariate analysis was performed to determine the independent prognostic factors. The results of this analysis confirmed that $\mathrm{PT}$ status and VEGF-C mRNA expression were independent relevant factors. Our results support Kimura's report and provide evidence at the mRNA level that VEGF-C expression is a significant prognostic factor both in univariate and multivariate analysis. Of the 92 patients in this study, recurrence was recognized in 65 patients $(70.7 \%)$ in the first 5 years after the operation. Thirty-four patients (52.3\%) developed a lymph node recurrence; 10 patients (15.4\%) developed lymph node and haematogenous recurrence. We suggest that the VEGF-C level in oesophageal cancer tissues might reflect formation of a metastatic focus through angiogenesis and lymphangiogenesis in the interstitium surrounding the primary tumour.

In conclusion, expression of VEGF-C is related to tumour invasion ( $\mathrm{pT})$, lymphatic invasion, lymph node metastasis ( $\mathrm{pN}$ ) and tumour stage (pTNM) in ESCC. The VEGF-C expression correlates with tumour metastatic recurrence in ESCC patients.

\section{Acknowledgments}

The authors have no financial affiliation or involvement with any commercial organization with direct financial interest in the subject or materials discussed in this manuscript. The authors would like to thank the Provincial Hospital Affiliated to Shandong University for its cooperation.

\section{References}

1. Zhang DW, Cheng GY, Huang GJ, et al. Operable squamous esophageal carcinoma: current results from the East. World J Surg 1994; 18: 347-54.

2. Watson A. Operable esophageal carcinoma: current results from the West. World J Surg 1994; 18: 361-6.

3. Mandard AM, Chasle J, Marnay J, et al. Autopsy findings in 111 cases of esophageal cancer. Cancer 1981; 48: 329-35.

4. Kitadai Y, Amioka T, Haruma K, et al. Clinicopathological significance of vascular endothelial growth factor (VEGF)-C in human esophageal squamous cell carcinomas. Int J Cancer 2001; 93: 662-6.

5. Kleespies A, Bruns CJ, Jauch KW. Clinical significance of VEGF-A, $-C$ and $-D$ expression in esophageal malignancies. Onkologie 2005; 28: 281-8.

6. Noguchi T, Takeno S, Shibata T, et al. VEGF-C expression correlates with histological differentiation and metastasis in squamous cell carcinoma of the esophagus. Oncol Rep 2002; 9: 99-9.

7. Korst RJ, Rusch VW, Venkatraman E, et al. Proposed revision of the staging classification for esophageal cancer. J Thorac Cardiovasc Surg 1998; 115: 660-70.

8. Chen G, Wang Z, Liu XY, Liu FY. Recurrence pattern of squamous cell carcinoma in the middle thoracic esophagus after modified Ivor-Lewis esophagectomy. World I Surg 2007; 31: 1107-14.

9. Liu XE, Sun XD, Wu JM. Expression and significance of VEGF-C and FLT-4 in gastric cancer. World J Gastroenterol 2004; 10: 352-5.

10. Mandriota SJ, Jussila L, Jeltsch $M$, et al. Vascular endothelial growth factor-C-mediated lymphangiogenesis promotes tumour metastasis. EMBO J 2001; 20: 672-82.

11. Noda E, Maeda K, Inoue T, et al. Predictive value of vascular endothelial growth factor-C expression for local recurrence of rectal carcinoma. Oncol Rep 2007; 17: 1327-31.

12. Korshunov A, Golanov A. The prognostic significance of vascular endothelial growth factor (VEGF C-1) immunoexpression in oligodendroglioma. An analysis of 91 cases. J Neurooncol 2000; 48: 13-19.

13. O-charoenrat P, Rhys-Evans P, Eccles SA. Expression of vascular endothelial growth factor family members in head and neck squamous cell carcinoma correlates with lymph node metastasis. Cancer 2001; 92: 556-68.

14. Fellmer PT, Sato K, Tanaka R, et al. Vascular endothelial growth factor-C gene expression in papillary and follicular thyroid carcinomas. Surgery 1999; 126: 1056-61.

15. Kajita T, Ohta Y, Kimura K, et al. The expression of vascular endothelial growth factor $C$ and its receptors in non-small cell lung cancer. Br J Cancer 2001; 85: 255-60. 
16. Kinoshita J, Kitamura K, Kabashima A, et al. Clinical significance of vascular endothelial growth factor-C (VEGF-C) in breast cancer. Breast Cancer Res Treat 2001; 66: 159-64.

17. Kabashima A, Maehara Y, Kakeji Y, Sugimachi K. Overexpression of vascular endothelial growth factor $C$ is related to lymphogenous metastasis in early gastric carcinoma. Oncology 2001; 60: 146-50.

18. Akagi K, Ikeda Y, Miyazaki M, et al. Vascular endothelial growth factor-C (VEGF-C) expression in human colorectal cancer tissues. Br J Cancer 2000; 83: 887-91.

19. Hashimoto I, Kodama J, Seki N, et al. Vascular endothelial growth factor- $\mathrm{C}$ expression and its relationship to pelvic lymph node status in invasive cervical cancer. $\mathrm{Br} J$ Cancer 2001; 85: 93-7.

20. Tsurusaki T, Kanda S, Sakai H, et al. Vascular endothelial growth factor-C expression in human prostatic carcinoma and its relationship to lymph node metastasis. Br J Cancer 1999; 80: 309-13.

21. Liu P, Chen W, Zhu H, et al. Expression of VEGF-C correlates with a poor prognosis based on analysis of prognostic factors in 73 patients with esophageal squamous cell carcinomas. Jpn J Clin Oncol 2009; 39: 644-50.

22. Chen G, Liu XY, Wang Z, Liu FY. Vascular endothelial growth factor C: the predicator of early recurrence in patients with N2 non-small-cell lung cancer. Eur J Cardiothorac Surg 2010; 37: 546-51.

23. Kimura $\mathrm{H}$, Kato $\mathrm{H}$, Tanaka $\mathrm{N}$, et al. Preoperative serum vascular endothelial growth factor-C (VEGF-C) levels predict recurrence in patients with esophageal cancer. Anticancer Res 2008; 28: 165-9 